\title{
然
}

Subdirección General de Estudios y Evaluación de Instrumentos

de Política Comercial*

\section{LA INVERSIÓN DIRECTA EXTRANJERA ANTE LA CRISIS SANITARIA}

La crisis sanitaria está causando profundos efectos humanos, sociales y económicos. En el ámbito de la inversión directa extranjera (IDE) se espera que resulte en descensos de los flujos entre el $30 \%$ y el $40 \%$ en los próximos años. En este artículo se examina la situación de la IDE, a la luz de las tendencias observadas en las últimas décadas en el mundo y en España, se analiza la relación entre estas tendencias y el impacto previsible de la crisis sanitaria, y se valoran las implicaciones para el diseño de las políticas relacionadas con la IDE.

Palabras clave: inversión directa extranjera, COVID-19, crisis sanitaria, atracción de inversiones, internacionalización.

Clasificación JEL: F01, F21.

\section{Introducción}

La pandemia COVID-19, originada por el coronavirus SARS-CoV-2, está causando profundos efectos humanos, sociales y económicos. En particular, en el ámbito económico, la extensión de la enfermedad y la necesaria adopción de medidas para contenerla están alterando radicalmente los escenarios previstos hace solo unos meses.

La extensión de la COVID-19 a nuevas áreas geográficas y la constatación de sus efectos dan lugar a continuas actualizaciones de las estimaciones de impacto, crecientemente pesimistas. Al mismo tiempo se genera el

* Secretaría de Estado de Comercio.

Versión de abril de 2020.

DOI: https://doi.org/10.32796/bice.2020.3122.6998 convencimiento de que solo una respuesta internacional coordinada podrá resultar efectiva para moderar sus efectos (OCDE, 2020).

La inversión directa extranjera (IDE) es una de las variables cuya evolución va a resultar afectada por la COVID-19. A principios de marzo, cuando la enfermedad se centraba en China y en otras economías del sudeste asiático, se estimaba que podría tener un efecto de reducción de la IDE entre el $5 \%$ y el $15 \%$, en los años 2020 y 2021, con relación a las previsiones anteriores. La preocupación se centraba en los efectos a través de las cadenas globales de valor en las que los países de esta región participan (UNCTAD, 2020a). A finales del mismo mes, con el epicentro de la pandemia ya localizado en Europa y extendiéndose hacia América y otras regiones, las estimaciones son de reducciones entre el $30 \%$ y el $40 \%$, y la $\triangleright$ 
preocupación alcanza no solo al funcionamiento de las cadenas globales de valor, sino también al efecto de la caída de la demanda global en los ingresos de las empresas multinacionales, los principales inversores internacionales (UNCTAD 2020b).

De hecho, la crisis sanitaria está ya produciendo una abrupta salida de flujos de capital de las economías emergentes, como ocurre habitualmente en momentos de fuerte aumento del riesgo (UNCTAD, 2020c). En muchos casos, estos países están teniendo que recurrir a apoyos del Fondo Monetario Internacional (FMI). A primeros de abril, 85 economías habían tenido que recurrir a financiación de emergencia del FMI; una cifra récord en la historia de la institución (Georgieva y Adhanom, 2020). Aunque estas alteraciones se centran en flujos financieros de naturaleza más volátil, es probable que acaben afectando también a la IDE, de forma que la crisis sanitaria impactará no solo en el volumen global de IDE, sino también en su distribución geográfica.

Se configura así un entorno complejo e incierto para la IDE, en el que no han desaparecido otras fuentes de riesgo, como las derivadas del Brexit, que, de hecho, están incidiendo ya en la inversión directa extranjera, al dar lugar a la relocalización de algunas empresas con sede en Reino Unido hacia la Unión Europea (UE), o de las tensiones entre los principales bloques comerciales.

En este marco, el objetivo de este artículo es examinar la situación de la IDE, a la luz de las tendencias observadas en las últimas décadas y de las explicaciones proporcionadas por la literatura económica. Mediante este análisis se pretende facilitar una mejor comprensión del papel que desempeña la IDE en la economía internacional, de las interrelaciones que genera entre distintas zonas geográficas y de la capacidad de reacción que pueden tener las políticas relacionadas con la IDE ante el escenario descrito.

En el epígrafe 2 se revisan algunas tendencias en la evolución de la IDE en el mundo, haciendo énfasis en su crecimiento desde los años setenta, en el aumento en paralelo de la apertura comercial y en los cambios que se han observado en su composición geográfica, que reflejan también cambios en el tipo de inversiones que se realizan y de agentes que la protagonizan.

En el epígrafe 3 se analizan los rasgos más destacables de la IDE en España, constatando cómo las tendencias globales han afectado también a la economía española. También se comprueba que España ha pasado de ser un país esencialmente receptor de IDE a ser una economía en la que las empresas domésticas están presentes, cada vez en mayor medida, en otros mercados a través de la inversión directa.

El epígrafe 4 se dedica a analizar algunas implicaciones de estas tendencias para el diseño de las políticas de inversión extranjera y a valorar la vigencia de este análisis ante la actual crisis sanitaria.

El artículo finaliza con un epígrafe de conclusiones.

\section{La inversión directa extranjera en el mundo: tendencias destacables}

En este epígrafe se revisan algunos rasgos de la evolución de la IDE en las últimas décadas, a la luz de las explicaciones que ha ido ofreciendo la literatura económica. Algunas de las referencias que se recogen se apoyan en la revisión, más exhaustiva, de dicha literatura realizada por Turrión (2009). 
La expansión de la IDE, a partir de la Segunda Guerra Mundial, suscitó el interés por conocer sus determinantes. Entre los enfoques más exitosos destaca el paradigma OLI (Dunning, 1980). De acuerdo con este enfoque, las empresas toman la decisión de invertir en el exterior atendiendo a un conjunto de factores que pueden agruparse en las tres categorías cuyo acrónimo en inglés da nombre al paradigma: propiedad (ownership), localización (location) e internalización (internalization).

Las ventajas de propiedad son un reflejo de los activos que posee la empresa, ya sean de naturaleza tangible o intangible, que puede explotar a través de la internacionalización. Las empresas podrían explotar estas ventajas de propiedad directamente (a través de la exportación o de la IDE) o indirectamente (mediante, por ejemplo, acuerdos, licencias o externalización de la producción). Para que la IDE tenga lugar, las ventajas de propiedad deben ir unidas a ventajas de internalización. La existencia de costes transaccionales (de negociación, supervisión y cumplimiento, entre otros) asociados a la explotación indirecta de estas ventajas puede determinar que su explotación directa resulte más rentable. Finalmente, las ventajas de localización hacen referencia al conjunto de factores que condicionan el interés de la empresa por localizar la producción en una determinada región (diferentes dotaciones factoriales, proximidad al mercado, costes de transporte, fiscalidad, etc.).

Las ventajas de propiedad pueden estar vinculadas a la existencia en las empresas de activos intensivos en conocimiento (como la tecnología o el know-how), con carácter de bien público. Este carácter de bien público determinaría también ventajas de internalización. Por esta vía se han asociado los modelos de capital intensivo en conocimiento al paradigma OLI, reforzando su fundamentación teórica (Markusen, 1998).

En las últimas décadas, los factores que posibilitaron el crecimiento de la IDE siguen cobrando fuerza. La globalización de la actividad económica se refleja en una intensificación de las relaciones entre los países, tanto a través del comercio de bienes y servicios como del movimiento de los factores productivos. El incremento de los flujos netos de IDE en el mundo y el aumento que, como resultado, se ha producido en el stock de IDE mundial son claros ejemplos de ello.

Así, el primer rasgo a destacar es la tendencia de crecimiento de los flujos de IDE, por encima del crecimiento del PIB (Gráfico 1 y Cuadro 1). Los flujos netos de IDE emitidos han pasado de representar cifras en torno al 0,6\% del PIB mundial en los años 70 y 80 a porcentajes del $1,4 \%$ en los 90 , del $2,3 \%$ en los 2000 y del 1,9\% entre 2010 y 2018.

Como resultado, se ha incrementado notablemente la relevancia que tiene el capital controlado por extranjeros en las economías domésticas del conjunto del mundo, lo que revela la profundidad que está alcanzando el proceso de globalización. Así, el stock de IDE acumulado, para el que la UNCTAD ofrece datos desde 1980, ha seguido una tendencia de fuerte crecimiento, pasando de representar el 5,5\% del PIB mundial en 1980 al $36,7 \%$ en 2018 . El peso de esta variable en relación al PIB mundial ha aumentado de forma prácticamente ininterrumpida, con algunas excepciones asociadas al ciclo económico o, en los últimos años, a decisiones políticas. Así, la repatriación de beneficios de las multinacionales de Estados Unidos, alentada por las reformas fiscales de 2017, diseñadas con este propósito, explica, en buena medida, la reducción de los flujos de IDE neta en 2018 , próxima al $13 \%$, que ha $\triangleright$ 


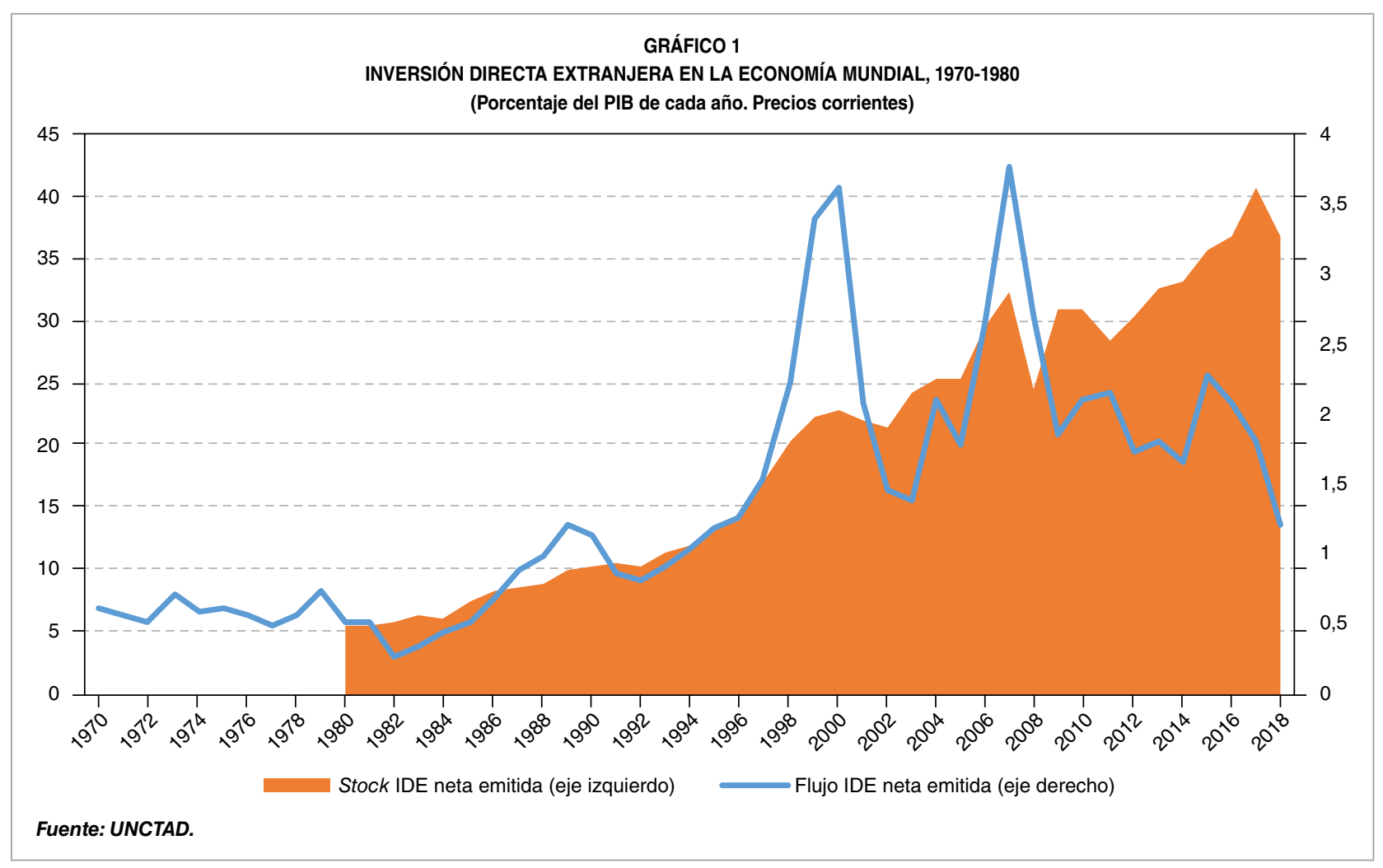

dado lugar también a una caída del stock de IDE mundial con relación al PIB (UNCTAD, 2019).

Esta tendencia al crecimiento de la IDE en las últimas décadas ha venido asociada a la expansión de los mercados mundiales y a la liberalización internacional, junto con la evolución y difusión de la tecnología y la reducción de costes de transporte. Estos cambios abren nuevas vías de aprovechamiento de las ventajas de propiedad y permiten explotar las ventajas de localización que ofrecen distintas zonas geográficas.

Teniendo en cuenta estos factores, las empresas podrían tomar decisiones de inversión directa en el extranjero con dos objetivos, no necesariamente excluyentes: producir en los mercados que desea abastecer (IDE horizontal) y optimizar costes, desplazando la producción hacia las localizaciones donde resulte más eficiente (IDE vertical). La IDE horizontal, favorecida por las ventajas de proximidad al $\triangle$

\section{CUADRO 1}

INVERSIÓN DIRECTA EXTRANJERA Y APERTURA AL COMERCIO DE BIENES EN EL MUNDO, 1970-1980 (Medias anuales del porcentaje del PIB. Precios corrientes)

\begin{tabular}{|l|c|c|c|}
\hline & Flujo IDE neta emitida & Stock IDE neta emitida & Exportaciones de bienes \\
\hline $1970-1979$ & 0,6 & - & 12,4 \\
$1980-1989$ & 0,6 & 7,2 & 15,0 \\
$1990-1999$ & 1,4 & 14,1 & 16,1 \\
$2000-2009$ & 2,3 & 25,8 & 21,3 \\
$2010-2018$ & 1,9 & 33,9 & 23,3 \\
\hline Fuente: UNCTAD. & &
\end{tabular}


mercado, la existencia de barreras al comercio exterior y elevados costes de transporte, podría tener un carácter sustitutivo del comercio internacional. Por el contrario, la IDE vertical, favorecida por la reducción de costes de transporte y de barreras al comercio, tendría un carácter más complementario.

En este sentido, un segundo rasgo destacable en la evolución de la IDE en las últimas décadas es que ha venido asociada a crecimientos de los flujos comerciales. Así, la relación positiva que se observa, al menos en los países desarrollados, entre el peso del stock de IDE, recibida o emitida, en el PIB e indicadores de la apertura del país al comercio de bienes sugiere que, entre 1980 y 2018, ha predominado este carácter complementario.

Esta relación es consistente con un papel significativo de la IDE vertical en el proceso de construcción de cadenas globales de valor, en especial desde los años noventa. Mediante la construcción de estas cadenas se optimizan los costes del sistema productivo, trasladando cada fase o eslabón de la producción donde resulta más eficiente. Esta búsqueda de eficiencia puede realizarse mediante contratos de aprovisionamiento o venta, licencias, otro tipo de acuerdos entre empresas o, cuando las ventajas de internalizar el proceso productivo se imponen, mediante inversión directa.

En el Gráfico 2 se presentan datos de los pesos en el PIB del stock de IDE recibida o emitida y de las exportaciones o importaciones de bienes en los distintos países de la OCDE (con excepción de Luxemburgo). Los datos reflejan el promedio anual del peso en el PIB de cada una de las variables en el periodo 1980-2018, o en el periodo para el que la $\triangle$

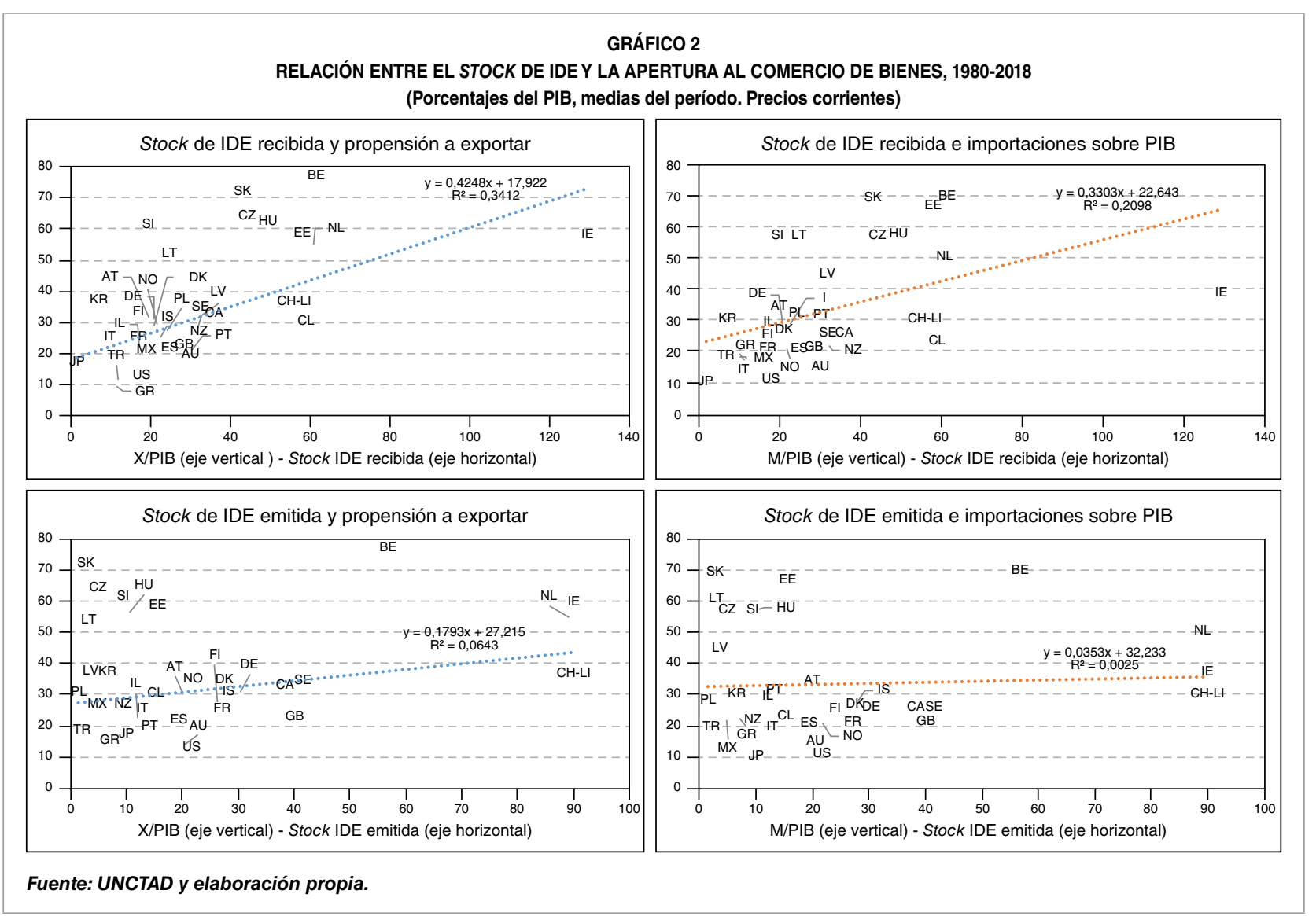


Subdirección General de Estudios y Evaluación de Instrumentos de Política Comercial

UNCTAD ofrece datos comparables para cada país (ver Cuadro 2).

El stock de IDE recibida en relación al PIB es un indicador de la importancia de los inversores extranjeros en la economía doméstica.
En los países de la OCDE, en el periodo analizado, este indicador guarda una relación positiva tanto con la propensión exportadora como con la participación de las importaciones en el PIB. Es decir, en el promedio del periodo, $\square$

CUADRO 2

CÓDIGOS PAÍS Y PERIODOS DE REFERENCIA

\begin{tabular}{|c|c|c|c|}
\hline \multirow{2}{*}{$\begin{array}{l}\text { Código } \\
\text { país }\end{array}$} & \multirow{2}{*}{ País } & \multicolumn{2}{|c|}{ Periodos de referencia para comparaciones con: } \\
\hline & & Stock de IDE recibida & Stock de IDE emitida \\
\hline$A U$ & Australia & $1980-2018$ & $1980-2018$ \\
\hline AT & Austria & $1980-2018$ & $1980-2018$ \\
\hline $\mathrm{BE}$ & Bélgica & $1980-2001$ y $2005-2018$ & $1980-2001$ у $2005-2018$ \\
\hline $\mathrm{CA}$ & Canadá & $1980-2018$ & $1980-2018$ \\
\hline $\mathrm{CL}$ & Chile & $1980-2018$ & $1980-2018$ \\
\hline $\mathrm{CZ}$ & República Checa & $1993-2018$ & $1993-2018$ \\
\hline DK & Dinamarca & $1980-2018$ & $1980-2018$ \\
\hline EE & Estonia & $1992-2018$ & $1992-2018$ \\
\hline $\mathrm{FI}$ & Finlandia & $1980-2018$ & $1980-2018$ \\
\hline $\mathrm{FR}$ & Francia & $1980-2018$ & $1980-2018$ \\
\hline $\mathrm{DE}$ & Alemania & $1990-2018$ & $1990-2018$ \\
\hline GR & Grecia & $1980-2018$ & $1986-2018$ \\
\hline $\mathrm{HU}$ & Hungría & $1990-2018$ & $1990-2018$ \\
\hline IS & Islandia & $1981-2018$ & $1985-2018$ \\
\hline IE & Irlanda & $1980-2018$ & $1985-2018$ \\
\hline IL & Israel & $1980-2018$ & $1980-2018$ \\
\hline IT & Italia & $1980-2018$ & $1980-2018$ \\
\hline$J P$ & Japón & $1980-2018$ & $1980-2018$ \\
\hline $\mathrm{KR}$ & Corea & $1980-2018$ & $1980-2018$ \\
\hline LV & Letonia & $1992-2018$ & $1992-2018$ \\
\hline LT & Lituania & $1992-2018$ & $1994-2018$ \\
\hline$M X$ & México & $1981-2018$ & $1980-2018$ \\
\hline NL & Países Bajos & $1980-2018$ & $1982-2018$ \\
\hline NZ & Nueva Zelanda & $1980-2018$ & $1980-2018$ \\
\hline NO & Noruega & $1980-2018$ & $1980-2018$ \\
\hline PL & Polonia & $1980-2018$ & $1981-2018$ \\
\hline PT & Portugal & $1980-2018$ & $1980-2018$ \\
\hline SK & Eslovaquia & $1993-2018$ & $1993-2018$ \\
\hline SI & Eslovenia & $1992-2018$ & $1992-2018$ \\
\hline ES & España & $1980-2018$ & $1980-2018$ \\
\hline SE & Suecia & $1980-2018$ & $1980-2018$ \\
\hline $\mathrm{CH}-\mathrm{LI}$ & Suiza y Liechtestein & $1983-2018$ & $1983-2018$ \\
\hline TR & Turquía & $1980-2018$ & $1985-2018$ \\
\hline GB & Reino Unido & $1980-2018$ & $1980-2018$ \\
\hline US & Estados Unidos & $1980-2018$ & $1980-2018$ \\
\hline
\end{tabular}


los países de la OCDE con mayor presencia de capital bajo control extranjero en relación con el tamaño de sus economías han sido también los más abiertos al comercio internacional, tanto en la vertiente exportadora como en la importadora.

El stock de IDE emitida en relación al PIB es indicativo de la importancia que para el país emisor tiene la presencia directa de sus empresas en el exterior. Aunque más débil que en el caso del stock de IDE recibida, también se observa una relación positiva entre este indicador, la propensión exportadora y la participación de las importaciones en el PIB. Este resultado indica que las economías desarrolladas, que en mayor medida han apostado por invertir en el exterior, tienden a ser también las más abiertas al comercio internacional.

En definitiva, los datos sugieren que en el periodo analizado, las economías desarrolladas cuyas empresas participan más en el comercio internacional han sido también aquellas para las que las empresas internacionalizadas a través de la IDE han tenido también mayor importancia.

La literatura económica revela que la capacidad de las empresas para internacionalizarse está relacionada con sus características intrínsecas y, en particular, con su productividad (Melitz, 2003). Las empresas que optan por la internacionalización son las más productivas y, dentro de estas, las que apuestan por la IDE en el exterior, frente a la exportación, son las que se sitúan en los estratos superiores de productividad (Helpman, 2004). Se trataría, además, de un fenómeno dinámico que genera un círculo virtuoso. Las empresas más productivas que se internacionalizan mejoran su estructura de costes y adquieren nuevos conocimientos que redundan en nuevas ganancias de productividad.
Estas aproximaciones deben ser matizadas ante el surgimiento, especialmente en las últimas décadas, de nuevos operadores de IDE, entre los que pueden citarse empresas multinacionales de países emergentes, multinacionales de propiedad estatal y fondos soberanos. Estos agentes están transformando la estructura geográfica de la IDE tradicional, favoreciendo su desconcentración hacia países en desarrollo.

En principio, no cabe esperar que las empresas de países en desarrollo (PED), en comparación con las de países desarrollados (PD), cuenten con grandes ventajas de propiedad (en el sentido del paradigma OLI) que favorezcan su transformación en empresas multinacionales a través de la IDE. No obstante, existen factores que pueden contribuir a explicar el fenómeno de las multinacionales de países emergentes. Entre ellos, cabe citar el papel de los agentes públicos, el incentivo que puede suponer la búsqueda de activos de conocimiento en el exterior y la ventaja competitiva que supone la familiaridad con entornos culturales e institucionales similares, y la aplicación de niveles tecnológicos parecidos y de estructuras organizativas equivalentes entre países de similar nivel de desarrollo (Durán y Álvarez, 2019). Un claro ejemplo de ello son las multilatinas, empresas originarias de algún país latinoamericano y con presencia en al menos otro país de la región (Casilda, 2018).

La estructura geográfica de la IDE ha reflejado estos cambios. La IDE ha dejado de ser un fenómeno entre PD, con cierta participación de otras áreas geográficas como receptoras, para adquirir una naturaleza más multipolar (Gráfico 3 y Cuadro 3).

En la década de los ochenta, los flujos de IDE se produjeron esencialmente entre PD que, en promedio anual, recibieron el $74,8 \% \triangleright$ 


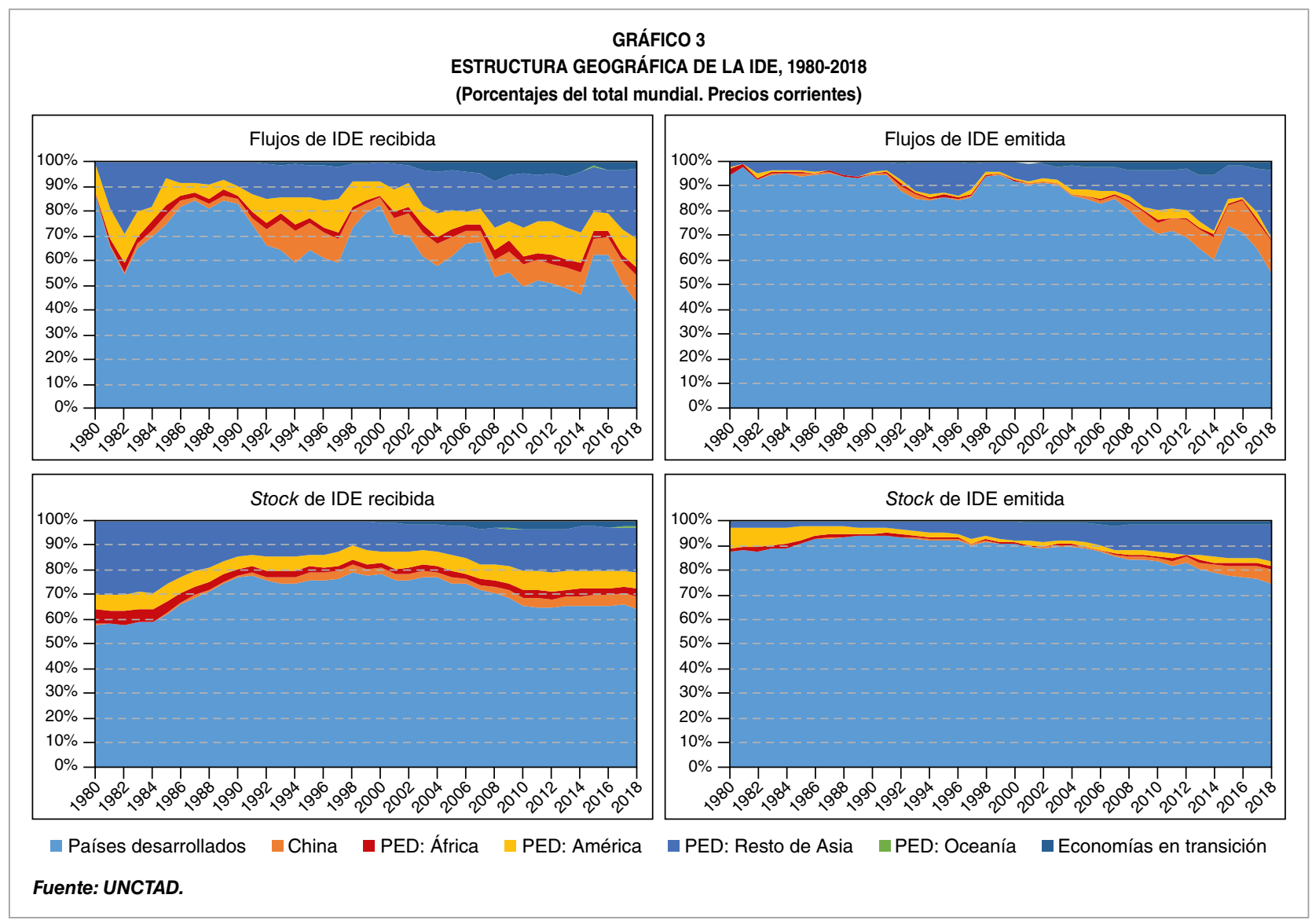

de la IDE neta y emitieron el 94,5\%. Los PED de Asia, incluyendo China, y de América mantenían cuotas significativas en la recepción de IDE (del $14,3 \%$ y el $8,2 \%$, respectivamente) y su contribución como emisores era más reducida (del $4,1 \%$ y el $0,7 \%$, respectivamente).

Entre 2010 y 2018, los PD recibieron tan solo el $51,7 \%$ de la IDE, en promedio anual, y emitieron el $66,9 \%$. Entre las economías en desarrollo receptoras no solo ganaron peso China (con una participación media del $8,4 \%$ y posicionada ya como segundo país receptor, tras EE UU), los PED del resto de Asia (21,5\%) y de América $(11,0 \%)$, sino también los de África $(3,2 \%)$ y el grupo de economías en transición (3,9\%).

Pero quizá lo más significativo es el peso que han ganado los PED como emisores de IDE. De una participación anual media del $4,5 \%$ en los ochenta han pasado a emitir el
33,1\% de los flujos mundiales de 2010 a 2018. Entre las áreas en desarrollo emisoras en este último periodo destacan China (8,8\%) y los PED del resto de Asia (17,9\%), con un papel también significativo de las economías en transición (3,4\%) y los PED de América (2,2\%).

Los cambios en los flujos de IDE neta se reflejan también en la estructura geográfica del stock de IDE. El stock de IDE recibida en los PED ha mantenido entre 2010 y 2018 un peso similar sobre el total mundial al que tenía en la década de los ochenta, aunque con algunos cambios en la composición interna, entre los que destaca el aumento de la importancia de China. Como emisores de IDE, el peso de los PED en el stock mundial ha aumentado desde el $9,7 \%$ hasta el $21,0 \%$, y China $(3,7 \%)$ y los PED del resto de Asia (12,8\%) cobran un creciente protagonismo. 
CUADRO 3

DISTRIBUCIÓN GEOGRÁFICA DE LA INVERSIÓN DIRECTA EXTRANJERA, 1980-2018

(Medias anuales de los porcentajes sobre el total. Precios corrientes)

\begin{tabular}{|c|c|c|c|c|c|c|c|}
\hline & \begin{tabular}{c|} 
Países \\
desarrollados
\end{tabular} & China & $\begin{array}{l}\text { PED: } \\
\text { África }\end{array}$ & $\begin{array}{c}\text { PED: } \\
\text { América }\end{array}$ & $\begin{array}{c}\text { PED: } \\
\text { Resto de Asia }\end{array}$ & PED: Oceanía & $\begin{array}{l}\text { Economías } \\
\text { en transición }\end{array}$ \\
\hline \multicolumn{8}{|c|}{ Flujo de IDE recibida } \\
\hline $1980-1989$ & 74,8 & 1,7 & 2,6 & 8,2 & 12,6 & 0,2 & 0,0 \\
\hline 1990-1999 & 68,4 & 7,9 & 1,9 & 9,1 & 11,7 & 0,1 & 1,0 \\
\hline $2000-2009$ & 64,8 & 6,9 & 2,9 & 7,9 & 13,8 & 0,1 & 3,6 \\
\hline $2010-2018$ & 51,7 & 8,4 & 3,2 & 11,0 & 21,5 & 0,1 & 3,9 \\
\hline \multicolumn{8}{|c|}{ Flujo de IDE emitida } \\
\hline $1980-1989$ & 94,5 & 0,4 & 0,7 & 0,7 & 3,7 & 0,0 & - \\
\hline $1990-1999$ & 89,0 & 0,8 & 0,6 & 1,1 & 8,3 & 0,0 & 0,4 \\
\hline $2000-2009$ & 85,8 & 1,5 & 0,3 & 1,6 & 8,9 & 0,0 & 1,9 \\
\hline $2010-2018$ & 66,9 & 8,8 & 0,7 & 2,2 & 17,9 & 0,0 & 3,4 \\
\hline \multicolumn{8}{|c|}{ Stock de IDE recibida } \\
\hline 1980-1989 & 63,4 & 0,5 & 4,5 & 6,3 & 25,1 & 0,1 & 0,1 \\
\hline 1990-1999 & 76,5 & 2,3 & 2,4 & 5,5 & 13,0 & 0,1 & 0,3 \\
\hline 2000-2009 & 74,4 & 2,5 & 2,4 & 6,1 & 12,5 & 0,0 & 2,0 \\
\hline $2010-2018$ & 65,2 & 4,1 & 2,9 & 7,2 & 17,6 & 0,1 & 2,9 \\
\hline \multicolumn{8}{|c|}{ Stock de IDE emitida } \\
\hline 1980-1989 & 90,3 & 0,1 & 1,3 & 5,7 & 2,6 & 0,0 & - \\
\hline 1990-1999 & 92,2 & 0,4 & 0,8 & 1,8 & 4,8 & 0,0 & 0,1 \\
\hline 2000-2009 & 87,8 & 0,6 & 0,5 & 1,6 & 8,3 & 0,0 & 1,2 \\
\hline $2010-2018$ & 79,0 & 3,7 & 0,8 & 2,2 & 12,8 & 0,0 & 1,5 \\
\hline
\end{tabular}

Estos cambios son más notables si se tiene en cuenta el aumento del peso de los flujos y el stock de IDE en relación al PIB mundial, ya analizado. En definitiva, las empresas de PED, especialmente asiáticos, internacionalizadas a través de la IDE, desempeñan ya un papel muy significativo en la economía mundial.

\section{La inversión directa extranjera en España}

Las tendencias observadas en la evolución de la IDE en el mundo se reflejan también en la evolución de la IDE en España.

Así, los flujos de IDE neta en España han seguido también una tendencia de crecimiento por encima del conjunto de la actividad económica, asociada tanto al ciclo económico como a factores políticos (Gráfico 4 y Cuadro 4). Esta tendencia ha dado como resultado un aumento de la importancia en nuestra economía de las empresas con participación directa extranjera, así como las empresas españolas con participaciones directas en el extranjero. Desde la literatura económica, ambos fenómenos se asocian a mejoras en la competitividad, tanto por las mejoras de capital físico, tecnológico y humano y por los efectos desbordamiento, tradicionalmente asociados a la IDE recibida, como por la vinculación entre la productividad empresarial y su proyección al exterior, vía IDE emitida (Esteve y Rodríguez, 2014a).

La IDE neta recibida, que representaba el $0,5 \%$ del PIB en los años setenta, registró una fase de fuerte crecimiento en los ochenta, $D$ 
Subdirección General de Estudios y Evaluación de Instrumentos de Política Comercial

posiblemente asociada al efecto positivo de la adhesión de España a la entonces Comunidad Económica Europea y al efecto de ampliación de mercado que ello suponía. El crecimiento fue aún más notable entre los últimos años noventa y los primeros del siglo veintiuno, llegando a alcanzar un peso en el PIB del $6,6 \%$ en el año 2000. En los años más recientes, entre 2010 y 2018, su peso se ha mantenido en promedio en el $2,1 \%$ del PIB.

El papel de España como emisor de IDE ha seguido también una tendencia ascendente, aunque con retraso respecto a la dinámica de la IDE recibida, asociada a la creciente integración de la economía española en cadenas globales de valor, así como a la internacionalización de grandes empresas españolas de diversos sectores de servicios e industrias de red, impulsada, entre otros factores, por procesos de liberalización interna. Así, es principalmente en los años noventa cuando los flujos de IDE emitida comienzan a ganar peso en el PIB en España. Desde mediados de esta década su importancia con relación al PIB ha sido de un orden de magnitud semejante o superior al de la IDE recibida. En el año 2000, la IDE neta emitida llegó a representar el 9,9\% del PIB y, entre 2010 y 2018, su peso promedio se ha situado en el $2,3 \%$ del PIB.

Como resultado de la evolución de los flujos de inversión, los stocks de IDE han observado también un fuerte crecimiento. Así, el stock $\triangleright$

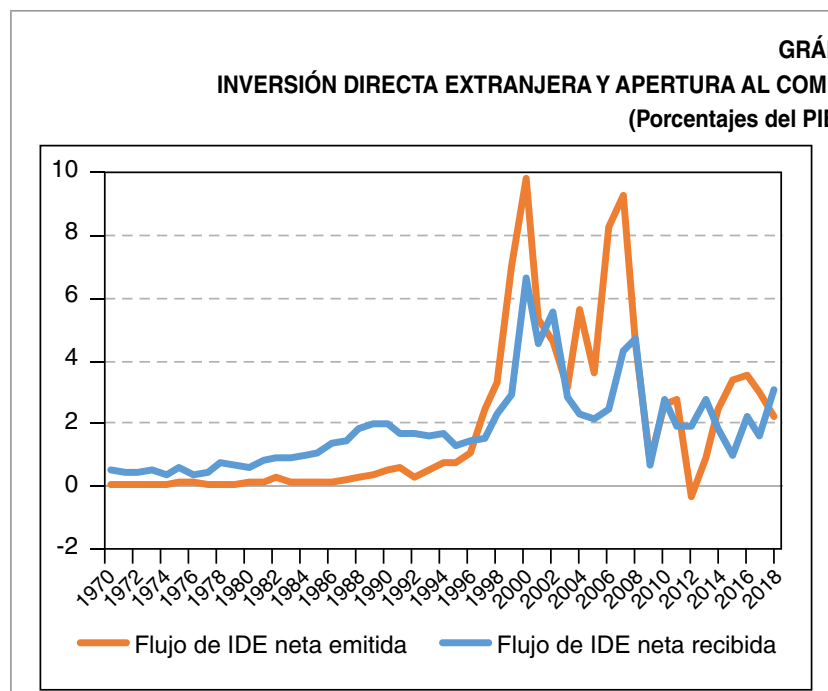

GRÁFICO 4

INVERSIÓN DIRECTA EXTRANJERA Y APERTURA AL COMERCIO DE BIENES EN LA ECONOMÍA ESPAÑOLA, 1970-1980 (Porcentajes del PIB. Precios corrientes)

Fuente: UNCTAD.

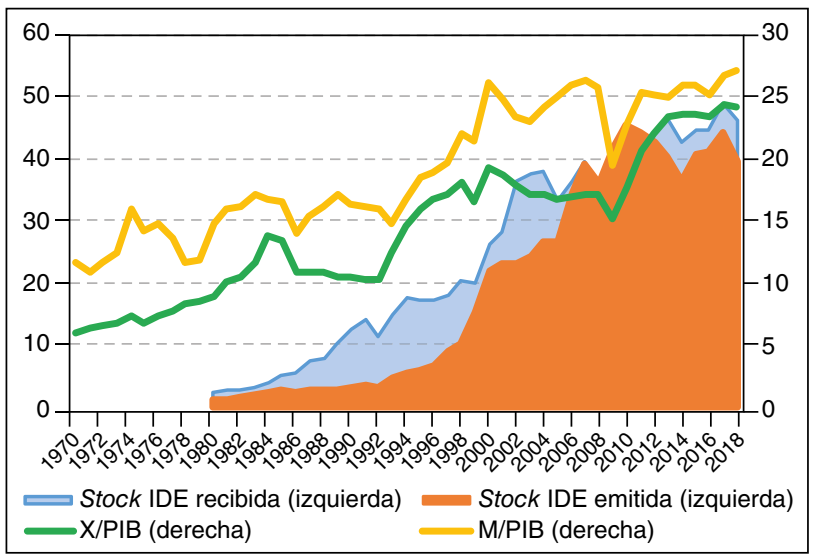

\section{CUADRO 4}

INVERSIÓN DIRECTA EXTRANJERA Y APERTURA AL COMERCIO DE BIENES EN ESPAÑA, 1970-1980 (Medias anuales del porcentaje del PIB. Precios corrientes)

\begin{tabular}{|l|c|c|c|c|c|}
\hline & $\mathbf{1 9 7 0 - 1 9 7 9}$ & $\mathbf{1 9 8 0 - 1 9 8 9}$ & $\mathbf{1 9 9 0 - 1 9 9 9}$ & $\mathbf{2 0 0 0 - 2 0 0 9}$ & $\mathbf{2 0 1 0 - 2 0 1 8}$ \\
\hline Flujo de IDE neta recibida & 0,5 & 1,2 & 1,8 & 3,6 & 2,1 \\
Flujo de IDE neta emitida & 0,1 & 0,2 & 1,7 & 5,5 & 2,3 \\
Stock IDE recibida & - & 5,0 & 16,3 & 35,4 & 45,0 \\
Stock IDE emitida & - & 2,0 & 6,6 & 29,7 & 41,7 \\
Exportaciones de bienes & 7,1 & 11,1 & 14,2 & 17,3 & 22,5 \\
Importaciones de bienes & 12,8 & 16,0 & 18,1 & 24,4 & 25,5 \\
\hline Fuente: UNCTAD. & \multicolumn{5}{|}{} \\
\hline
\end{tabular}


de IDE recibida en relación al PIB, que es indicativo de la importancia de empresas de capital extranjero en nuestra economía, ha pasado de cifras ligeramente superiores al $5 \%$ en la década de los ochenta al $45 \%$ entre 2010 y 2018. Similarmente, la importancia relativa que tiene la proyección de nuestras empresas en el exterior a través de la inversión directa ha crecido desde el 2,0\% en los ochenta al 41,7\% del PIB entre 2010 y 2018.

Esta evolución ha sido paralela a un aumento de la apertura comercial al exterior, tanto en la vertiente de las exportaciones como en la de las exportaciones. Aproximando esta apertura a través del comercio de bienes, se observan aumentos de la propensión exportadora, desde el $7,1 \%$ en los ochenta hasta el $22,5 \%$ entre 2010 y 2018. La participación de las importaciones en el PIB creció desde el 12,8\% al 25,5\% en los mismos periodos de referencia.

Es destacable, además, que en los últimos años, los posteriores a la recesión económica, la distancia entre las participaciones de las importaciones y las exportaciones de bienes en el PIB se ha reducido con relación a periodos anteriores. Esta evolución del comercio de bienes, junto al superávit que tradicionalmente registra la balanza de servicios española, ha contribuido a que 2019 sea el octavo año consecutivo de crecimiento económico con superávit de balanza de bienes y servicios y capacidad de financiación frente al exterior (Subdirección General de Estudios y Evaluación de Instrumentos de Política Comercial, 2020). Uno de los factores que puede haber contribuido a este resultado, que favorece la sostenibilidad del crecimiento económico, es la mejora de competitividad asociada al comportamiento de la IDE recibida y emitida.

En la evolución de la IDE en España destacan algunos rasgos de su distribución geográfica. Entre ellos, la progresiva pérdida del peso relativo de la IDE procedente de la UE, frente a otras regiones que ganan importancia relativa y el notable peso de la IDE emitida hacia América Latina. Estos rasgos son indicativos de la integración de empresas españolas en la economía mundial a través de cadenas globales de valor que enlazan no solo con los países europeos, sino también, y de forma directa, con otras áreas geográficas.

Los datos del Registro de Inversiones Exteriores permiten ilustrar estos rasgos para el periodo 1993-2019, cuando la IDE se analiza en términos de flujo, y 2007-2018, para el análisis de los stocks. Se ha optado por realizar el análisis de flujos en términos brutos y, para el caso de la IDE recibida, atendiendo al país último de origen de la inversión. Se evita así la imagen distorsionada que deriva de la vehiculización de inversiones a través de países intermedios, bien por motivos fiscales o de organización de las empresas multinacionales, que figuran como los de origen inmediato de estos flujos. Asimismo, el análisis se realiza descontando las operaciones tipo ETVE (Entidades de Tenencia de Valores Extranjeros), es decir, operaciones de transmisiones de participaciones en empresas extranjeras dentro del mismo grupo empresarial (con independencia de que se lleven a cabo o no por entidades acogidas al régimen fiscal de ETVE) y operaciones llevadas a cabo por ETVE cuando su actividad es exclusivamente la tenencia de valores extranjeros.

En el Gráfico 5 y el Cuadro 5 se observan los flujos de IDE bruta emitidos y recibidos por España. En relación con la IDE recibida, destaca la progresiva pérdida de importancia relativa de la UE, frente a áreas como América del Norte y China, y resto de Asia y Oceanía.

En relación a la IDE emitida, destacan la presencia de América Latina a lo largo de todo el periodo, el aumento de peso en las dos $\triangleright$ 


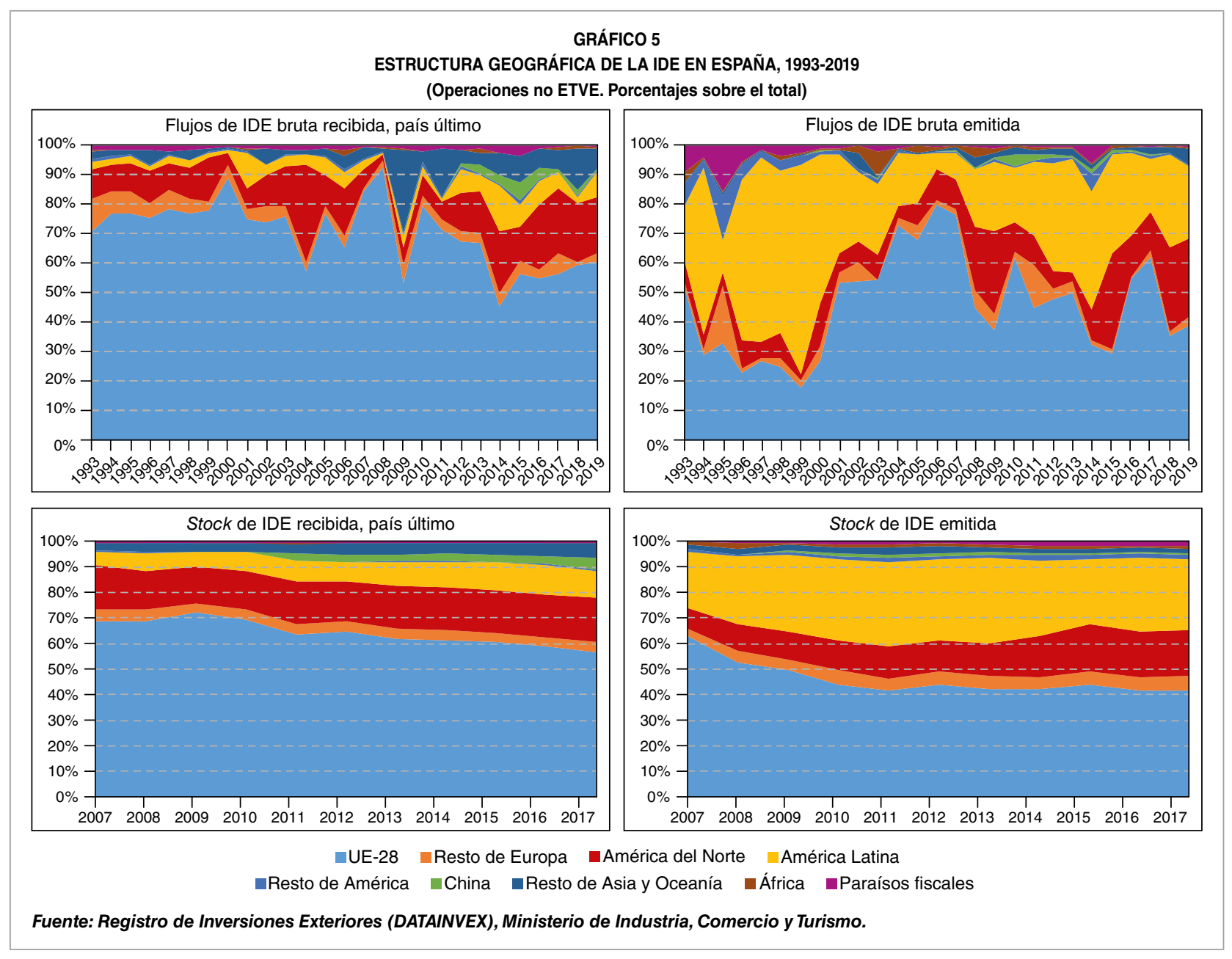

últimas décadas de América del Norte y, en la década más reciente, de China y el resto de Asia y Oceanía. Algunos estudios empíricos sobre la IDE realizada por las empresas manufactureras españolas sugieren que estas adoptan estrategias de inversión horizontal en países avanzados y de inversión vertical en los de menos nivel de desarrollo (Núñez y otros, 2011), por lo que la inversión observada hacia los PED de América Latina y Asia podría estar asociada a la búsqueda de mejoras de eficiencia y optimización de costes en diversas fases de la producción.

El análisis de los stocks de IDE recibida y emitida confirma la progresiva pérdida de importancia de la UE a favor de otras regiones.
Así, las empresas de la UE han pasado de representar el 66,9\% del stock de IDE recibida en España (medido por la posición neta inversora) al $62,1 \%$ entre 2010 y 2017 . Entre las regiones que han ganado peso como emisoras de IDE hacia España destacan China, que de una participación marginal ha pasado a representar el 2,5\% del stock entre 2010 y 2018, y América Latina, con un aumento de peso de 3,3 puntos porcentuales entre los periodos tomados como referencia.

La distribución geográfica del stock de IDE emitida revela también cambios notables. La participación de la UE como receptora de las inversiones de nuestras empresas es más reducida y también decreciente en los últimos $\triangleright$ 
CUADRO 5

ESTRUCTURA GEOGRÁFICA DE LA IDE EN ESPAÑA

(Operaciones no ETVE, porcentajes sobre el total)

\begin{tabular}{|c|c|c|c|c|c|c|c|c|c|}
\hline & UE-28 & $\begin{array}{l}\text { Resto de } \\
\text { Europa }\end{array}$ & $\begin{array}{l}\text { América } \\
\text { del Norte }\end{array}$ & $\begin{array}{l}\text { América } \\
\text { Latina }\end{array}$ & $\begin{array}{l}\text { Resto de } \\
\text { América }\end{array}$ & China & $\begin{array}{c}\text { Resto de } \\
\text { Asia y } \\
\text { Oceanía }\end{array}$ & África & $\begin{array}{c}\text { Paraísos } \\
\text { fiscales }\end{array}$ \\
\hline \multicolumn{10}{|c|}{ Flujos de IDE bruta recibida (país último) } \\
\hline 1993-1999 & 76,3 & 6,5 & 10,6 & 2,0 & 0,6 & 0,0 & 2,2 & 0,1 & 1,5 \\
\hline $2000-2009$ & 74,5 & 3,5 & 11,0 & 4,3 & 0,4 & 0,0 & 5,0 & 0,4 & 1,0 \\
\hline 2010-2019 & 61,9 & 3,6 & 15,6 & 6,4 & 0,7 & 2,1 & 7,9 & 0,5 & 1,2 \\
\hline \multicolumn{10}{|c|}{ Flujos de IDE bruta emitida } \\
\hline 1993-1999 & 29,4 & 4,5 & 6,0 & 47,0 & 5,5 & 0,0 & 0,5 & 1,0 & 6,1 \\
\hline 2000-2009 & 56,8 & 3,7 & 11,9 & 22,3 & 0,8 & 0,3 & 1,3 & 2,1 & 0,9 \\
\hline $2010-2019$ & 45,7 & 3,5 & 15,4 & 29,4 & 1,2 & 1,1 & 2,1 & 0,6 & 1,0 \\
\hline \multicolumn{10}{|c|}{ Stock de IDE recibida (país inmediato) } \\
\hline 2007-2009 & 69,9 & 4,2 & 15,7 & 6,0 & 0,2 & 0,0 & 3,2 & 0,3 & 0,5 \\
\hline $2010-2017$ & 62,1 & 4,0 & 16,5 & 9,3 & 0,4 & 2,5 & 4,4 & 0,3 & 0,5 \\
\hline \multicolumn{10}{|c|}{ Stock de IDE emitida } \\
\hline 2007-2009 & 55,1 & 3,9 & 9,9 & 26,1 & 0,7 & 0,4 & 1,9 & 1,6 & 0,4 \\
\hline $2010-2017$ & 42,6 & 5,2 & 15,0 & 30,2 & 1,5 & 0,9 & 2,3 & 0,9 & 1,4 \\
\hline
\end{tabular}

años, alcanzando un peso promedio del $42,6 \%$ entre 2010 y 2017 en el stock de IDE emitida. Es destacable el peso de América Latina como destino prioritario. Entre 2010 y 2017, el 30,2\% del stock de IDE emitida por empresas españolas corresponde a esta área geográfica. América del Norte es la tercera gran área hacia la que se proyectan las empresas españolas mediante IDE, mientras que la participación en el stock de China y el resto de Asia y Oceanía es aún reducida, aunque presenta un crecimiento incipiente.

Conjuntamente, el crecimiento de la IDE recibida y emitida y su diversificación geográfica apuntan a una creciente integración de la economía española en la economía mundial, no solo a través de la UE, sino también mediante relaciones directas con otras áreas geográficas. Un dato destacable es la importancia de América Latina, no solo como área hacia la que se orienta la IDE de las empresas situadas en España, sino también como emisora de IDE hacia España. Estos datos sugieren que España podría estar actuando como plataforma para filiales «trampolín» en la expansión de las multinacionales europeas hacia América Latina y de las multilatinas en su expansión hacia Europa (Pla-Barber y otros, 2019).

\section{Algunas implicaciones para las políticas de inversión exterior}

El análisis de los epígrafes precedentes revela que la IDE, en el mundo y en España, estaba ya sujeta a profundos cambios antes de la irrupción de la pandemia. Estos cambios estaban determinando modificaciones en las políticas de inversión extranjera y, en ocasiones, estaban originados por ellas.

Así, la UNCTAD señala que, en el año 2018, 55 economías introdujeron 112 medidas relativas a la inversión extranjera y que más de un tercio de estas medidas supusieron $\triangleright$ 
restricciones a la inversión. Estas restricciones se fundamentan, principalmente, en preocupaciones relacionadas con la relación entre la seguridad nacional y la propiedad extranjera de infraestructuras críticas o tecnologías clave. Los mecanismos de notificación y supervisión también están ganando importancia, como respuesta a estas preocupaciones (UNCTAD, 2019).

En cierta medida, esta orientación restrictiva se asocia a la aparición de nuevas áreas geográficas y de nuevos agentes en el panorama de inversores internacionales. En particular, el protagonismo de fondos soberanos y empresas multinacionales de titularidad pública, cuyas actuaciones pueden responder a lógicas distintas de las de mercado, intensifica las preocupaciones relativas a la seguridad nacional, especialmente cuando estos agentes proceden de áreas geopolíticas menos afines. Los fondos soberanos se articulan en torno a cuatro grandes polos: Noruega (con un fondo soberano vinculado al sistema público de pensiones), Oriente Medio, China, y el sudeste asiático. Entre las empresas multinacionales de titularidad pública tienen especial importancia las de algunas economías de la UE, China, Malasia, India, Sudáfrica y Emiratos Árabes (Durán y Álvarez, 2019).

Otro factor clave en la prospectiva de la IDE, presente antes del brote del COVID-19, es la percepción de síntomas de desaceleración o incluso de retraimiento de inversiones vinculadas a la construcción de las cadenas globales de valor. Esta dinámica responde, por un lado, al progresivo agotamiento de las oportunidades de mejoras de eficiencia, dado el amplio despliegue inversor realizado en años anteriores $y$, por otro lado, al enfrentamiento entre grandes bloques comerciales y la bilateralización de las relaciones comerciales internacionales. Se configura así un entorno menos estable, en el que las decisiones políticas, a veces de naturaleza geoestratégica, pueden generar distorsiones económicas que afecten a la rentabilidad de las inversiones internacionales. En consecuencia, cabe esperar que los inversores deseen minimizar este tipo de riesgos, reorientándose hacia las economías domésticas o hacia las regiones o países menos susceptibles de resultar afectados por estas decisiones. En definitiva, reconstruyendo las cadenas globales de valor para dotarlas de mayor robustez.

No obstante, recibir inversiones continúa siendo una prioridad. Los países diseñan incentivos fiscales para atraer inversiones extranjeras (o, como en el caso de EE UU, para repatriarlas), reducen barreras y cargas administrativas, aplican regímenes específicos a través de zonas económicas especiales y renuevan o firman nuevos acuerdos de protección de inversiones. En 2018 se tenía constancia de cerca de 5.400 zonas económicas especiales, en 147 países, en desarrollo o desarrollados, frente a unas 4.000 cuatro años antes, y se firmaron cuarenta nuevos acuerdos de protección de inversiones, frente a los veinticuatro que llegaron a su término (UNCTAD, 2019).

En este contexto, las agencias de promoción de inversiones están evolucionando, de una orientación más general, basada en la eliminación de barreras y la difusión de información a potenciales inversores, al enfoque hacia determinados tipos de inversiones, acordes con las prioridades económicas y tecnológicas de los países receptores. Las agencias más evolucionadas se orientan, además, hacia la IDE sostenible (Gugler, 2019).

La irrupción de la pandemia COVID-19 puede acelerar estos procesos. Las interrupciones de suministros ocasionadas por las medidas $\square$ 
adoptadas para su contención son un argumento adicional para que las empresas multinacionales deseen contar con cadenas de producción más robustas y seguras. Las dificultades para obtener equipos médicos y sanitarios para combatir la enfermedad darán lugar a que la seguridad sanitaria se contemple, como un aspecto más de la seguridad nacional, para restringir inversiones extranjeras en empresas de sectores clave (o, aunque pueda parecer contradictorio, para fomentar la inversión extranjera en esos mismos sectores, como forma de impulsar la autosuficiencia, cuando la IDE se lleva a cabo mediante inversiones de nueva planta y no a través de simples adquisiciones de empresas preexistentes). El impacto de la crisis sanitaria en las bolsas puede abrir la puerta a inversiones oportunistas frente a las que los Gobiernos están ya intensificando mecanismos de supervisión y control de las inversiones extranjeras.

En este sentido, en el ámbito de la UE, se aprobó en marzo de 2019 el Reglamento 2019/452 para el control de las inversiones extranjeras directas en la Unión. Este reglamento persigue armonizar la aplicación de los instrumentos de los que disponen los Estados miembros para controlar inversiones directas, procedentes de Estados no integrados en el Espacio Económico Europeo, que puedan afectar a la seguridad o al orden público y establecer un mecanismo de cooperación entre los Estados miembros.

Al amparo del Reglamento 2019/452, el Real Decreto Ley $8 / 2020$ ha modificado el modelo de control de inversiones extranjeras, introduciendo en determinados supuestos y en ámbitos sensibles autorizaciones previas. El Real Decreto Ley 11/2020, a su vez, ha introducido nuevas modificaciones en este régimen. Aunque estas medidas responden a la urgencia de la situación, son también ilustrativas del creciente grado de preocupación por disponer de mecanismos de supervisión y control adecuados de la IDE extranjera.

En la labor de atracción de inversiones exteriores, el impacto económico de la pandemia también va a configurar un entorno más exigente. Los descensos estimados de la IDE para 2020 y 2021, y la conveniencia de contar con flujos de inversión para reactivar las economías, supondrán una competencia más intensa por atraerlas. En el contexto descrito de reconfiguración y, posiblemente, de acortamiento de las cadenas globales de valor, es previsible que los entornos percibidos como más seguros y estables refuercen sus ventajas competitivas.

En este sentido, España cuenta con algunas fortalezas notables. Su integración en la UE y la sujeción al exigente marco comunitario que ello conlleva son elementos que refuerzan la confianza y potencian la atracción de inversiones orientadas al mercado europeo, tanto para inversores de la UE como de terceros países. La vigencia de 76 Acuerdos de Protección Recíproca de Inversiones entre España y terceros países actúa en el mismo sentido.

El acceso a un gran mercado es, precisamente, una de las ventajas de localización que perciben los inversores extranjeros en España (ICEX-Invest In Spain y otros, 2019). Estos inversores valoran muy positivamente la posición española en algunas áreas que consideran también como las más relevantes para las decisiones de inversión. Entre estas áreas figuran el tamaño del mercado (que puede identificarse con el mercado comunitario), el capital humano o las dotaciones de infraestructuras. Por otro lado, los inversores extranjeros identifican ámbitos, como la reducción de cargas administrativas, la flexibilidad de horarios laborales $\triangleright$ 
o el manejo de idiomas, en los que sería conveniente seguir mejorando.

Otra fortaleza es el origen de la IDE recibida en España, vinculado principalmente a países y regiones con los que tenemos fuertes vínculos políticos (especialmente, con los países de la UE), económicos e incluso culturales. A título ilustrativo, incluso en un entorno internacional más complejo, España seguirá siendo percibida como una plataforma interesante para acceder al mercado comunitario por parte de las empresas multilatinas. $Y$ es probable que las empresas multinacionales europeas que deseen ampliar actividades a América Latina lo continúen haciendo también a través de inversiones en España.

Una fortaleza adicional es que la labor que realiza la Dirección de Invest in Spain de ICEX está ya enfocada hacia la atracción de inversiones que respondan a las prioridades económicas del país. Así, su primera línea de acción estratégica es la atracción de proyectos de inversión directa extranjera de los países, sectores y negocios con mayor crecimiento potencial en España.

Esta labor se complementa con otros instrumentos ya desarrollados que buscan facilitar la inversión exterior, en particular, en conexión con la incorporación de talento a nuestra economía. Por ejemplo, a través del Programa de Residencia para Inversores y Emprendedores se apoya la movilidad internacional de inversores, emprendedores y profesionales altamente cualificados, para facilitar la entrada de inversión extranjera y de talento.

Finalmente, la complejidad del entorno internacional para la IDE, agravada por los efectos económicos de la pandemia, no debería hacer olvidar la necesidad de potenciar la internacionalización de las empresas españolas, también a través de la IDE, como vía para mejorar su competitividad. El desarrollo de los programas de apoyo e instrumentos financieros que persiguen este objetivo debería continuar siendo una prioridad para que el sector exterior contribuya a la recuperación y al crecimiento económico a largo plazo.

\section{Conclusiones}

La crisis sanitaria global tendrá profundos efectos en muchos ámbitos de la economía y la IDE no será una excepción.

Lo flujos y stocks de IDE han registrado fuertes crecimientos en las últimas décadas, asociados a la apertura de las economías, la liberalización de mercados y los cambios tecnológicos. Sin embargo, ya en los últimos años, la irrupción de nuevos agentes, las tensiones comerciales y geopolíticas y el agotamiento del despliegue de las cadenas globales de valor se percibían como factores de riesgo para el crecimiento futuro de la IDE.

España no ha sido ajena a esta dinámica, tanto como receptora como, de forma destacada, emisora de IDE. Se ha percibido un fuerte crecimiento de ambos flujos de inversión $y$, como resultado, una presencia creciente del capital extranjero en nuestra economía y de las empresas españolas en otros mercados. La distribución geográfica también ha variado, aunque la UE, América del Norte y América Latina continúan siendo nuestros socios más destacados.

Es previsible que los efectos económicos de la crisis sanitaria intensifiquen algunas de las tensiones que ya condicionaban el desarrollo futuro de la IDE, al tiempo que aumentará la necesidad de los países por atraer flujos de inversión que coadyuven a la reactivación económica. 
España tampoco será ajena a este proceso. La pertenencia a la Unión Europea, que garantiza el acceso a su mercado y un entorno institucional estable, y la capacidad de reacción de los instrumentos de atracción de inversiones y de apoyo a la internacionalización se configuran como fortalezas que será preciso continuar reforzando.

\section{Bibliografía}

Casilda Béjar, R. (2018). Las empresas multilatinas. Boletín Económico de ICE (3105). https://doi. org/10.32796/bice.2018.3105.6624

Dunning, J. H. (1973). The determinants of international production. Oxford Economic Papers, 25(3), 289-336

Dunning, J. H. (1980). Toward an eclectic theory of international production: Some empirical results. Journal of International Business Studies, 11(1), 9-31.

Durán, J., y Álvarez, I. (2019). Expansión internacional de las empresas multinacionales. Estructura y naturaleza institucional. ICE, Revista de Economía (909). https://doi.org/10.32796/ice.2019. 909.6898

Esteve, S., y Rodríguez, D. (2014a). Características de las empresas que realizan IED. Empresas multipaís y multiproducto. En R. Myro (Director) y M. E. Álvarez López (Coordinadora), España en la Inversión directa internacional. Instituto de Estudios Económicos. Colección Estudios.

Esteve, S., y Rodríguez, D. (2014b). Efectos de la inversión exterior sobre la exportación, el capital humano y el esfuerzo tecnológico. En R. Myro (director) y M. E. Álvarez López (coordinadora), España en la Inversión directa internacional. Instituto de Estudios Económicos. Colección Estudios.

Georgieva, K., y Adhanom Ghebreyesus, T. (3 de abril de 2020). Some say there is a trade-off: save lives or save jobs - this is a false dilema. The Telegraph.

Gugler, P. (2019). Recent policy developments regarding foreign investment. ICE, Revista de Economía (909). https://doi.org/10.32796/ice.2019. 909.6892

Helpman, E., Melitz, M. J., \& Yeaple, S. R. (2004). Export versus FDI with heterogeneous firms. American Economic Review, 94(1).

ICEX-Invest in Spain (2019). Multinacionales por marca España e International Center for Competitiveness del IESE. Barómetro del clima de negocios en España desde la perspectiva del inversor extranjero. Resultados 2019.

Markusen, J. R. (1998). Multinational Firms, Location and Trade, World Economy, 21(6).

Melitz, M. J. (2003). The impact of trade on intra-industry reallocation and aggregate industry productivity. Econometrica, 71(6).

Núñez, J. A., Turrión, J., y Velázquez, F. J. (2011). Decisiones de internacionalización y heterogeneidad empresarial: el caso de las manufacturas españolas. Cuadernos Económicos de ICE, 1(82). https://doi.org/10.32796/cice.2011.82.6025

OECD (2020). OECD Economic Outlook, Interim Report March 2020, OECD Publishing. https:// doi.org/10.1787/7969896b-en

Pla-Barber, J., Villar, C., y Botella, A. (2019). La expansión interregional en lberoamérica: la creación de valor a través de filiales trampolín. ICE, Revista de Economía (909). https://doi.org/ 10.32796/ice.2019.909.6894

Real Decreto Ley 8/2020, de 17 de marzo, de medidas urgentes extraordinarias para hacer frente al impacto económico y social del COVID-19. Boletín Oficial del Estado, n.․ 73, de 18 de marzo de 2020. https://www.boe.es/eli/es/rdl/2020/ $03 / 17 / 8$

Real Decreto Ley 11/2020, de 31 de marzo, por el que se adoptan medidas urgentes $\square$ 
Subdirección General de Estudios y Evaluación de Instrumentos de Política Comercial

complementarias en el ámbito social y económico para hacer frente al COVID-19. Boletín Oficial del Estado, n.o 91, de 1 de abril de 2020. https:// www.boe.es/eli/es/rdl/2020/03/31/11

Reglamento (UE) 2019/452 del Parlamento Europeo y el Consejo, de 19 de marzo de 2019, para el control de las inversiones extranjeras directas en la Unión. Diario Oficial de la Unión Europea, n.. 79, de 21 de marzo de 2019.

Subdirección General de Estudios y Evaluación de Instrumentos de Política Comercial (2020). El comercio exterior ante los retos de 2020. Boletín Económico de ICE (3121). https://doi.org/ 10.32796/bice.2020.3121.6983

Turrión, J. (2009). La decisión de internacionalización de las empresas: un modelo teórico con inversión horizontal y vertical. Estudios de la Fundación, Serie Tesis. FUNCAS.

UNCTAD (2019). World Investment Report 2019. Special Economic Zones.

UNCTAD (2020a). Global Investment Trend Monitor, n. ${ }^{\circ} 34$ [Special Coronavirus Edition] (UNCTAD/ DIAE/IA/INF/2020/2).

UNCTAD (2020b). Global Investment Trend Monitor, n. ${ }^{\circ} 35$ [Special Coronavirus Edition] (UNCTAD/ DIAE/IA/INF/2020/3).

UNCTAD (2020c). Trade and Development Report Update. The Covid-19 Shock to Developing Countries: Towards a «whatever it takes» programme for the two-thirds of the world's population being left behind. https://unctad.org/en/PublicationsLibrary/gds_tdr2019_covid2_en.pdf 